\title{
Outpatient treatment for uterine polyps
}

A long overdue break with traditional practice, backed up by solid evidence

\author{
Jan Bosteels consultant ${ }^{12}$, Steven Weyers professor of gynaecology ${ }^{3}$ \\ ${ }^{1}$ Department of Obstetrics and Gynaecology, Imelda Hospital, Bonheiden, Belgium; ${ }^{2}$ Centre for Evidence-Based Medicine, Belgian Branch of the \\ Dutch Cochrane Centre, 3000 Leuven, Belgium; ${ }^{3}$ Department of Obstetrics and Gynaecology, Ghent University Hospital, Ghent, Belgium
}

\begin{abstract}
Abnormal uterine bleeding is one of the most common reasons why women present to hospital gynaecology departments. Even in developed countries such as Belgium ${ }^{1}$ a large proportion of these women are still being offered traditional dilatation and curettage combined with hysteroscopy under general anaesthesia, a costly approach that is inconvenient as it involves an overnight stay in hospital. The dominance of traditional inpatient dilatation and curettage is currently being challenged most recently by Cooper and colleagues in the linked study (doi:10.1136/bmj.h1398) evaluating outpatient diagnosis and treatment for women with uterine polyps, a common cause of intrauterine bleeding. ${ }^{2}$
\end{abstract}

During dilatation and curettage the operator is unable to see inside the uterine cavity, and tissue is therefore removed in a blind manner. The procedure is usually done to rule out endometrial cancer, and although the diagnostic accuracy for detecting endometrial cancer is high it is moderate for other endometrial disease, such as uterine polyps. ${ }^{3}$ Between $20 \%$ and $40 \%$ of women with abnormal uterine bleeding have polyps. Hysteroscopy allows the operator to visualise the entire uterine cavity, investigate the underlying causes of bleeding, and remove tissue under direct vision.

Over the past few decades advances in technology have allowed the development of small calibre endoscopes that can be used without anaesthesia in an outpatient setting. It is now feasible to perform outpatient hysteroscopies under local anaesthesia both for diagnosis and for therapeutic procedures such as the removal of endometrial polyps. ${ }^{45}$ Treatment can be done at the moment of diagnosis using the "see and treat" principle. This approach has many potential benefits for women and for health systems, including faster diagnosis and treatment of abnormal bleeding and the avoidance of hospital admission. Therefore the position of the traditional dilatation and curettage for diagnosis and treatment of abnormal uterine bleeding has become questionable. ${ }^{6}$

Cooper and colleagues' trial (Outpatient Polyp Treatment, OPT) is the first large multicentre prospective randomised controlled trial evaluating the clinical effectiveness of outpatient versus inpatient polyp removal by hysteroscopy. ${ }^{2}$ Previous studies reported an improvement in patients' symptoms after removal of polyps by hysteroscopy in the outpatient versus inpatient setting ${ }^{7}$ along with good patient tolerability, low pain scores, and faster recovery after outpatient treatment. ${ }^{8}$

In the OPT trial women were recruited from 31 outpatient hysteroscopy clinics in UK National Health Service hospitals: $72 \%$ of women allocated to outpatient polypectomy were treated in "see and treat" clinics. The primary outcome was women's own appreciation of their bleeding pattern at six months.

The authors should be applauded for prioritising a patient reported outcome, and for setting a clear example for future researchers to follow. Many in this specialty still fail to understand the importance of patient reported outcomes, which is regrettable from the viewpoint of the women who participate in trials, policy makers who use the results to inform decisions, and health systems that operate inefficiently without data on outcomes that matter to patients. ${ }^{9}$ The COMET (Core Outcome Measures in Effectiveness Trials) Initiative, which supports the development, reporting, and adoption of a core outcomes set, may help in this regard. ${ }^{1011}$

The potential advantages of outpatient treatment for polyps include the avoidance of general anaesthesia, immediate treatment after diagnosis, a half day's absence from work, and not having to rely on others for transport after the procedure. However, the limitations of performing intrauterine surgery in a conscious patient may well outweigh all these apparent benefits. The merit of the OPT trial ${ }^{2}$ lies in clearly determining the limits of this trade-off. The trial shows that outpatient treatment is non-inferior to traditional inpatient treatment for alleviating uterine bleeding. Women treated as outpatients were, however, on average twice as likely to have only partial or even failed polyp removals and needed further gynaecological surgery significantly more often than controls treated as inpatients.

Although women treated as outpatients had higher mean pain scores than controls, only a few women in both arms found their treatment unacceptable. More than $90 \%$ of women in both arms would recommend either procedure to friends or relatives. In qualitative interviews the women treated as outpatients reported that the pain experienced in the short term was off-set by the 
convenience of a fast relief from their disturbed quality of life in the longer term. Four women experienced serious adverse events (2\%); all had been treated as inpatients (2\%). A full economic evaluation of the OPT trial was an integral component from the outset: its findings will be published at a later date.

The body of evidence on the effectiveness, safety, feasibility, and acceptability of outpatient removal of polyps for treating abnormal uterine bleeding is now on solid enough ground to justify establishing outpatient hysteroscopy units so women can at least have a choice. Technological improvements and a desire to facilitate rapid recovery and discharge should make outpatient, ambulatory, or office based interventions more widespread, in Europe and elsewhere.

The uptake of outpatient hysteroscopy in Belgium is at this moment seriously hampered by an obsolete reimbursement system. As women's healthcare providers it is our responsibility to discuss the implications of the available evidence with policy makers of healthcare systems across Europe and worldwide. For Belgian women, the findings and conclusions of the OPT trial have come at a critical moment, as discussions have recently begun to encourage a break with tradition in favour of evidence. Perhaps change is finally on the way.

Competing interests: We have read and understood the BMJ policy on declaration of interests and declare the following: none.
Provenance and peer review: Commissioned; not externally peer reviewed.

1 National Institute for Health and Disability Insurance. Health care department, NIHDI, Belgium.

2 Cooper NAM, Clark TJ, Middleton L, Diwakar L, Smith P, Denny E, et al. Outpatient versus inpatient uterine polyp treatment for abnormal uterine bleeding: randomised controlled non-inferiority study. BMJ 2015;350:h1398.

3 Clark TJ, Voit D, Gupta JK, Hyde C, Song F, Khan KS. Accuracy of hysteroscopy in the diagnosis of endometrial cancer and hyperplasia: a systematic quantitative review. JAMA 2002;288:1610-21.

4 Marwah V, Bhandari SK. Diagnostic and interventional microhysteroscopy with use of the coaxial bipolar electrode system. Fertil Steril 2003;79:413-7.

5 Cicinelli E, Tinelli R, Loiudice L, Loiudice I, Francavilla M, Pinto V. Office polypectomy without anesthesia with Alphascope: a randomized controlled study. J Minim Invasive Gynecol 2010;17(6 Suppl 1).

6 Emanuel MH, Wamsteker K, Lammes FB. Is dilatation and curettage obsolete for diagnosing intrauterine disorders in premenopausal patients with persistent abnormal uterine bleeding? Acta Obstet Gynecol Scand 1997;76:65-8.

7 Clark TJ, Godwin J, Khan KS, Gupta JK. Ambulatory endoscopic treatment of symptomatic benign endometrial polyps. A feasibility study. Gynecol Endosc 2002;11(2-3):91-7.

8 Marsh FA, Rogerson LJ, Duffy SRG. A randomized controlled trial comparing outpatient versus day case endometrial polypectomy. BJOG 2006;113:896-901.

9 Greenhalgh J, Long AF, Flynn R. The use of patient reported outcome measures in routine clinical practice: lack of impact or lack of theory? Soc Sci Med 2005;60:833-43.

10 Williamson PR, Altman DG, Blazeby JM, Clarke M, Gargon E. The COMET (Core Outcome Measures in Clinical Effectiveness Trials) initiative. Trials 2011;12(Suppl 1):A70.

11 Gargon E, Gurung B, Medley N, Altman DG, Blazeby JM, Clarke M, et al. Choosing important health outcomes for comparative effectiveness research: a systematic review. PLoS One 2014;9:e99111.

Cite this as: BMJ 2015;350:h1469

(c) BMJ Publishing Group Ltd 2015 